\title{
Comparing the Cost Effectiveness of a Celiac Disease Panel to a Testing Cascade
}

\author{
Caleb Bazyler ${ }^{1, *}$, Kevin Breuel ${ }^{2}$ \\ ${ }^{1}$ Department of Sport, Exercise, Recreation, and Kinesiology, East Tennessee State University, Johnson City, TN, USA \\ ${ }^{2}$ Department of Obstetrics, and Gynecology, East Tennessee State University Clinical Laboratories, \\ Quillen College of Medicine, Johnson City, TN, USA \\ *Corresponding author: bazyler@etsu.edu
}

\begin{abstract}
Recent reductions in healthcare funding in the United States has pressured clinical laboratories to provide the same quality of diagnostic testing with fewer resources. Testing cascades have been developed to assist in the diagnosis of various illnesses, which use fewer tests and subsequently reduce costs. However, the cost effectiveness of a celiac disease (CD) testing cascade compared to a panel is currently unknown. Therefore, the purpose of this study was to determine if a $\mathrm{CD}$ testing cascade was equivalent to a panel in identifying patients deemed likely for $\mathrm{CD}$, and to compare their cost effectiveness in a sample of symptomatic patients from Northeast Tennessee. A retrospective analysis using a CD testing cascade was performed on 933 outpatient samples referred to our laboratory from 2012 to 2017 with a request for a celiac disease serology panel. The seroprevalence of CD for the panel and the cascade were the same in this population $(1.82 \%, 95 \%$ binomial confidence interval: $1.06 \%$ to $2.90 \%$ ). The total cost of the CD cascade was $268 \%$ less than the cost of the panel resulting in a savings of $\$ 44,705$, which translates to a savings of $\$ 47.92 /$ patient. Based on these findings, we recommend utilization of the cascade to identify patients with likely $\mathrm{CD}$. In the future, creative use of novel testing strategies can have significant contributions to healthcare reform and afford patients more cost-effective clinical diagnostic testing.
\end{abstract}

Keywords: healthcare reform, seroprevalence, celiac disease cascade

Cite This Article: Caleb Bazyler, and Kevin Breuel, "Comparing the Cost Effectiveness of a Celiac Disease Panel to a Testing Cascade." International Journal of Celiac Disease, vol. 6, no. 1 (2018): 11-13. doi: 10.12691/ijcd-6-1-6.

\section{Introduction}

Celiac disease (CD) is an immune-mediated inflammatory reaction to dietary gluten which results in injury to the mucosa of the small intestine with a loss of absorptive surface area $[1,2]$. The seroprevalence of $\mathrm{CD}$ in the general population is between $0.8-1 \%[1,3,4]$ and is on the rise. Despite rising diagnosis rates $[1,4,5], \mathrm{CD}$ remains underdiagnosed in the United States [1]. Currently, the favored strategy for increasing detection of $\mathrm{CD}$ is an active-case finding approach involving serological testing of patients with symptoms (e.g. abdominal pain, diarrhea, chronic fatigue) or conditions (e.g. irritable bowel syndrome, Down syndrome, Turner syndrome) closely associated with CD. Among adult patients with chronic abdominal symptoms, tissue transglutaminase (TTG IgA) and endomysial antibodies (EMA IgA) have the highest accuracy for diagnosis of $\mathrm{CD}$. In IgA deficient patients, TTG IgG, EMA IgG or deamidated gliadin peptides (DGP IgG) are measured to screen for possible CD. Despite advancements in serological testing for $\mathrm{CD}$, small intestinal biopsy with histology remains the gold-standard for diagnosing CD. Considering the invasiveness and expense of intestinal biopsies, serological testing cascades have been developed to identify patients who are at a higher risk for $\mathrm{CD}$.
Recent healthcare reform has led to the progressive contraction of healthcare funding [6,7]. As a result, clinical laboratories have been pressured "to do more with less" to provide the same quality of diagnostic testing with fewer resources. Historically, laboratories have used testing cascades to assist in diagnosis of various illnesses, which use fewer tests and subsequently reduce costs. While use of cascades for various illnesses (e.g. bacterial infections [8], hypercholesterolaemia [9], sepsis [10]) have been shown to save money, physicians often order a testing panel consisting of multiple assays. Various testing cascades currently exist for $\mathrm{CD}$ from national clinical laboratories (Mayo medical laboratories, ARUP, LabCorp); however, their cost effectiveness compared to a $\mathrm{CD}$ panel is currently unknown. Therefore, the purpose of this study was to determine if a $\mathrm{CD}$ testing cascade was equivalent to a panel in identifying patients deemed likely for $\mathrm{CD}$, and to compare their cost effectiveness in a sample of symptomatic patients from Northeast Tennessee.

\section{Materials and Methods}

\subsection{Study Sample}

The patient population consisted of 933 consecutive outpatient samples $($ male $=299$, female $=634$, age $=45.06 \pm$ 
19.84) referred to our laboratory from January 1st, 2012 to August 1st, 2017 with a request for a celiac serology panel to be performed. Samples were analyzed at East Tennessee State University (ETSU) Clinical Laboratories, Quillen College of Medicine. The ETSU Institutional Review Board (IRB) determined that the study did not meet the definition of human subjects' research and therefore did not require IRB review and approval.

\subsection{Laboratory Analyses}

Immunoglobulin A testing was performed on an automated chemistry analyzer (Beckman Coulter AU 480, Bellport, NY) using the immunoturbidimetric IgA assay (Kamiya Biomedical Company, Tukwila, WA). The CD assays: TTG IgA, DGP IgA, TTG IgG, DGP IgG were performed on a Phadia 250 Immunoassay Analyzer (assays and analyzer purchased from Fisher Scientific, Hampton, NH). The EMA IgA test was performed at Laboratory Corporation of America (Burlington, NC).

The CD panel included the following assays: IgA, TTG IgA, EMA IgA, DGP IgA, DGP IgG. In our subsequent retrospective analysis we applied the results from the panel to a cascade we developed based in principle on Mayo clinical laboratories $\mathrm{CD}$ cascade (Figure 1). For the $\mathrm{CD}$ cascade, samples with $\operatorname{IgA} \geq 7 \mathrm{mg} / \mathrm{dL}(\mathrm{n}=933)$ were applied to TTG IgA and were further grouped based on the package insert reference ranges: TTG $\operatorname{IgA}>10 \mathrm{U} / \mathrm{mL}$, positive; 7-10 U/mL, equivocal; $<4 \mathrm{U} / \mathrm{mL}$, negative. Patient samples with $\mathrm{IgA}<7 \mathrm{mg} / \mathrm{dL}$ were removed from the analyses due to the small sample size $(n=8)$.

\subsection{Statistical Analyses}

Prevalence rates for positive serology are expressed as a percentage of the total number of patient samples with 95\% binomial confidence intervals (CIs) based on the criteria used by Katz et al., [4]. Namely, a positive TTG IgA or equivocal TTG IgA with either positive EMA IgA or DGP IgA were deemed likely for CD. Reimbursements for the various tests were obtained from the 2017 Medicare Clinical Diagnostic Laboratory Fee Schedule for Tennessee. Grand total cost for the CD panel and cascade were compared to assess cost effectiveness. Analyses were conducted using Microsoft Excel 2013 (Microsoft Corporation, Redmond, WA, USA).

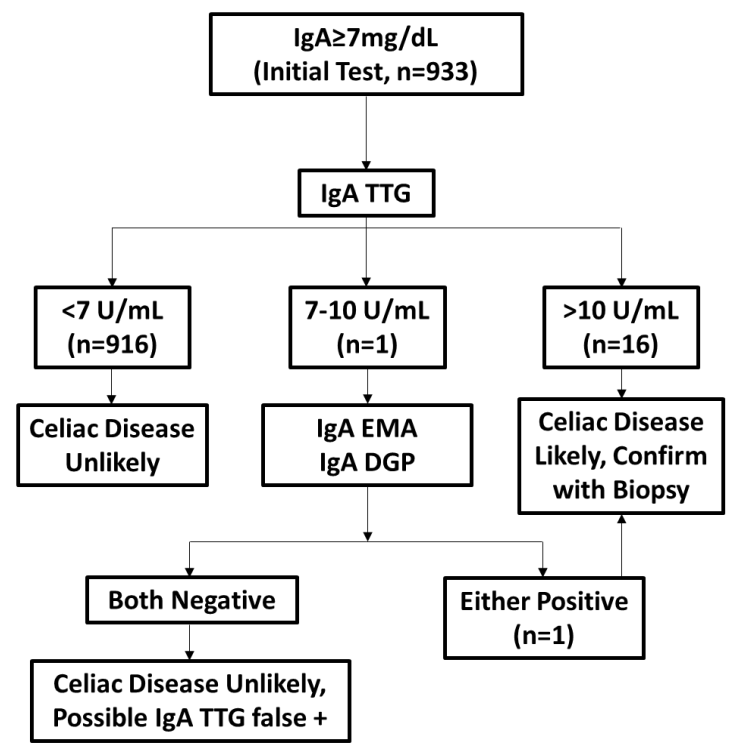

Figure 1. CD Testing Cascade with results

\section{Results}

Overall the seroprevalence of CD in this population of patients from Northeast Tennessee was 1.82\% (95\% CI: $1.06 \%$ to $2.90 \%$ ) with 17 of 933 positive samples. The CD cascade successfully captured all patients deemed likely for $\mathrm{CD}$. The EMA IgA results for 1 patient sample were positive with all other tests negative, thus this patient was considered unlikely for CD. The seroprevalence of CD in pediatric ( $<18 \mathrm{yr}, 2$ of 99$)$ and adult samples $(>18 \mathrm{yr}, 15$ of 834) was similar. Therefore, the results from both of these groups were combined for further analyses. The total cost of the CD cascade was $268 \%$ less than the cost of the panel resulting in a savings of $\$ 44,705$ (Table 1). Furthermore, the panel cost $\$ 76.42 /$ patient, whereas the cascade cost $\$ 28.50 /$ patient, resulting in a savings of $\$ 47.92 /$ patient.

Table 1. Cost analysis for CD panel vs. cascade

\begin{tabular}{|c|c|c|c|c|c|c|}
\hline \multirow[b]{2}{*}{ Assay } & \multicolumn{3}{|c|}{ Panel } & \multicolumn{3}{|c|}{ Cascade } \\
\hline & Number of tests & *Cost/assay & Total Cost & Number of tests & *Cost/assay & Total Cost \\
\hline $\operatorname{IgA}$ & 933 & $\$ 12.76$ & $\$ 11,905$ & 933 & $\$ 12.76$ & $\$ 11,905$ \\
\hline IgA TTG & 933 & $\$ 15.71$ & $\$ 14,657$ & 933 & $\$ 15.71$ & $\$ 14,657$ \\
\hline IgA DGP & 933 & $\$ 15.71$ & $\$ 14,657$ & 1 & $\$ 15.71$ & $\$ 15.71$ \\
\hline IgG DGP & 933 & $\$ 15.71$ & $\$ 14,657$ & 0 & $\$ 15.71$ & $\$ 0.00$ \\
\hline IgA EMA & 933 & $\$ 16.53$ & $\$ 15,422$ & 1 & $\$ 16.53$ & $\$ 16.53$ \\
\hline Total Cost & \multicolumn{3}{|c|}{$\$ 71,300$} & \multicolumn{3}{|c|}{$\$ 26,595$} \\
\hline
\end{tabular}

*Cost/assay determined from 2017 Medicare Clinical Diagnostic Laboratory Fee Schedule for Tennessee.

\section{Discussion}

The purpose of this study was to determine if a CD testing cascade was equivalent to a panel in identifying patients deemed likely for $\mathrm{CD}$, and to compare their cost effectiveness in a sample of symptomatic patients from Northeast Tennessee. Our results showed that the CD cascade captured all patients with a high probability of CD and did so at a much lower cost compared to the panel. The seroprevalence of $\mathrm{CD}$ in the present study $(1.82 \%)$ was slightly greater than that reported for the general population $(0.8 \%$ to $1.0 \%)[1,3,4]$. This is likely because the present study only tested symptomatic patients. Also, it is important to note that IgA deficient samples 
$(<7 \mathrm{mg} / \mathrm{dL})$ were not included in this analysis and may have influenced the observed seroprevalence rate.

Recent reductions in healthcare funding has pressured clinical laboratories to provide the same quality of diagnostic testing with fewer resources. The findings from the current study support the use of a CD cascade that resulted in lower costs, but still captured all patients with a high probability of $\mathrm{CD}$. In support of our findings, unpublished data from Mayo Clinical Laboratories also determined that a $\mathrm{CD}$ cascade resulted in a savings of $\$ 39,527$ and a diagnosis rate of $87 \%$ compared to a hospital-ordered panel over a 12-month period [11]. Cascades used for other illnesses have also been effective at reducing costs while maintaining diagnosis rates. For example, a family screening strategy for hypercholesterolemia resulted in a cost savings of $\sim \$ 13,638 /$ case detected compared to a universal screening method in the United Kingdom [9]. To further illustrate the impact on healthcare spending, if the current $\mathrm{CD}$ cascade was used instead of the panel in 1,000 clinical laboratories across the United States it would result in a cost savings of nearly $\$ 40$ million. Considering the rising CD diagnosis rates and hospital discharges for celiac disease $[1,12]$, reducing healthcare spending through the use of cascades is paramount. Future research should address the utility and cost benefit of cascades compared to other panels used to screen for relatively common diseases.

\section{Conclusions}

The seroprevalence of CD for the panel and the cascade were the same $(1.82 \%)$ in a sample of symptomatic patients from Northeast Tennessee over a 4.5-year period. However, the CD cascade resulted in a $\$ 47.92$ savings/patient compared to the panel. Based on these findings, we recommend utilization of the cascade to identify patients with likely CD. In the future, creative use of novel testing strategies can have significant contributions to healthcare reform and afford patients more cost-effective clinical diagnostic testing.

\section{Acknowledgements}

We would like to thank the ETSU Clinical Laboratory staff for their assistance with conducting the assays.

\section{Statement of Competing Interests}

The authors have no competing interests.

\section{References}

[1] Rubio-Tapia, A.; Hill, I.D.; Kelly, C.P.; Calderwood, A.H.; Murray, J.A. "Acg clinical guidelines: Diagnosis and management of celiac disease." Am J Gastroenterol, 108 (5), 656-676. 2013.

[2] Lebwohl, B.; Rubio-Tapia, A.; Guandalini, S.; Newland, C.; Assiri, A. "Diagnosis of celiac disease." Gastrointest Endosc Clin N Am, 22 (4), 661-677. 2012.

[3] Fasano, A.; Berti, I.; Gerarduzzi, T.; Not, T.; Colletti, R.B.; Drago, S.; Elitsur, Y.; Green, P.H.; Guandalini, S.; Hill, I.D., et al. "Prevalence of celiac disease in at-risk and not-at-risk groups in the united states: A large multicenter study." Arch Intern Med, 163 (3), 286-292. 2003.

[4] Katz, K.D.; Rashtak, S.; Lahr, B.D.; Melton, L.J., 3rd; Krause, P.K.; Maggi, K.; Talley, N.J.; Murray, J.A. "Screening for celiac disease in a north american population: Sequential serology and gastrointestinal symptoms." Am J Gastroenterol, 106 (7), 13331339. 2011

[5] Catassi, C.; Kryszak, D.; Bhatti, B.; Sturgeon, C.; Helzlsouer, K.; Clipp, S.L.; Gelfond, D.; Puppa, E.; Sferruzza, A.; Fasano, A. "Natural history of celiac disease autoimmunity in a usa cohort followed since 1974." Ann Med, 42 (7), 530-538. 2010.

[6] Lippi, G.; Bassi, A.; Bovo, C. "The future of laboratory medicine in the era of precision medicine." J Lab Precis Med. 1 (7). 1-5. 2016.

[7] Lippi, G.; Plebani, M. "Laboratory medicine does matter in science (and medicine)... yet many seem to ignore it." Clin Chem Lab Med, 53 (11), 1655-1656. 2015.

[8] Tang, H.; Huang, T.; Jing, J.; Shen, H.; Cui, W. "Effect of procalcitonin-guided treatment in patients with infections: A systematic review and meta-analysis." Infection, 37 (6), 497-507. 2009.

[9] Marks, D.; Wonderling, D.; Thorogood, M.; Lambert, H.; Humphries, S.E.; Neil, H.A. "Cost effectiveness analysis of different approaches of screening for familial hypercholesterolaemia." $B M J$ 324 (7349), 1303. 2002.

[10] Wilke, M.H.; Grube, R.F.; Bodmann, K.F. "The use of a standardized pct-algorithm reduces costs in intensive care in septic patients - a DRG-based simulation model." Eur J Med Res, 16 (12), 543-548. 2011

[11] Snyder, M.R. "Unpublished mayo clinic data." 2012.

[12] Borrelli, E.P. "Trends in hospitalizations for celiac disease in the United States.” Int J Celiac Dis, 5 (4), 150-154. 2017. 